\title{
Répercussions des antiviraux à action directe contre l'hépatite $C$ sur les taux d'hospitalisation au Canada, de 2012 à 2016
}

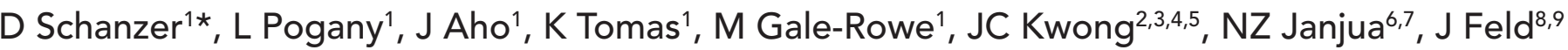

\section{Résumé}

Contexte : Au Canada, les hospitalisations liées aux infections par le virus de l'hépatite C (VHC) et aux hépatopathies ont connu une augmentation moyenne de 6,0\% par année entre 2004 et 2010 , et les projections établies en 2010 prévoyaient une hausse additionnelle de $4 \%$ jusqu'en 2016. Cependant, la première génération d'antiviraux à action directe (AAD) est entrée sur le marché en 2012 et des AAD de deuxième génération, à la fois efficaces et bien tolérés, ont été homologués au Canada en 2014. Les répercussions de ces médicaments sur le fardeau canadien des maladies associées au VHC n'ont pas été documentées.

Objectif : Évaluer les répercussions potentielles des AAD sur le fardeau de la maladie : a) en comparant les projections de référence faites en 2010 aux réels taux d'hospitalisations liées aux infections par le VHC et aux hépatopathies suivant l'introduction d'AAD au Canada; et b) en documentant l'adoption des traitements contre le VHC qui est associée à ces taux.

Méthodologie : Les dossiers d'hospitalisation des patients ayant reçu un diagnostic d'infection chronique par le VHC ou d'hépatopathie chronique ont été obtenus de la Base de données sur les congés des patients (BDCP) du Canada et classés par exercice financier pour 2004 à 2016. Le nombre réel d'hospitalisations a été comparé aux projections de référence en fonction de l'année et de la cohorte de naissance (cohortes divisées en tranches de cinq ans) chez les patients nés entre 1925 et 1989. Le nombre de nouvelles ordonnances émises chaque mois pour les traitements contre le VHC a été obtenu de la base de données CDH CompuScript d'IQVIA (anciennement IMS Health); ces données ont ensuite été colligées par année et par groupe d'âge, et comparées aux tendances en matière d'hospitalisations.

Résultats : Comparativement aux projections de référence, les taux d'hospitalisations ont connu une légère baisse en 2014-2015 et en 2015-2016, ce qui été suivi par une baisse plus importante en 2016-2017 (32\% sous les valeurs prédites; intervalle de confiance [IC] à $95 \%$ : 27 à $37 \%$ ). Les baisses les plus importantes ont été observées chez les patients nés avant 1960 (c.-à-d. âgés de 55 ans et plus), chez qui le taux d'hospitalisation réel était inférieur de $40 \%$ aux valeurs prédites pour 2016-2017. Le nombre de nouvelles ordonnances pour un traitement contre le VHC a augmenté de 5484 en 2012 à 2013, atteignant un maximum de 17775 en 2015-2016. Le nombre de nouvelles ordonnances correspond à environ 1,3 fois le nombre d'hospitalisations observé en 2012 à 2013 et à 5 fois le nombre d'hospitalisations pour 2015-2016.

Conclusions : Au Canada, après une augmentation significative du taux d'adoption des AAD de deuxième génération observée en 2015, les taux d'hospitalisations liées aux infections par le VHC et aux hépatopathies ont connu une légère baisse. Le fardeau de la maladie demeure toutefois lourd. Les bases de données administratives sur la santé créées pour surveiller le fardeau des maladies dans la nouvelle ère thérapeutique devraient fournir plus de données en permettant de faire des liens entre les antécédents thérapeutiques, l'évolution de la maladie et les résultats individuels.

Citation proposée : Schanzer D, Pogany L, Aho J, Tomas K, Gale-Rowe M, Kwong JC, Janjua NZ, Feld J. Répercussions des antiviraux à action directe contre l'hépatite $C$ sur les taux d'hospitalisation au Canada, de 2012 à 2016. Relevé des maladies transmissibles au Canada 2018;44(7/8):167-74. https://doi.org/10.14745/ccdr. v44i78a01f

Mots-clés : hépatite $C$, fardeau de la maladie, tendances, hospitalisation, surveillance des répercussions des AAD

\section{Affiliations}

${ }^{1}$ Centre de la lutte contre les maladies transmissibles et les infections, Agence de la santé publique du Canada, Ottawa (Ontario)

${ }^{2}$ Institut de recherche en services de santé, Toronto (Ontario)

${ }^{3}$ Santé publique Ontario, Toronto (Ontario)

${ }^{4}$ Département de médecine familiale et communautaire, Université de Toronto, Toronto (Ontario)

${ }^{5}$ École de santé publique Dalla Lana, Université de Toronto, Toronto (Ontario)

${ }^{6}$ Centre de contrôle des maladies de la Colombie-Britannique, Vancouver (Colombie-Britannique)

${ }^{7}$ École de la santé publique et des populations, Université de la Colombie-Britannique, Vancouver (Colombie-Britannique)

${ }^{8}$ Toronto Centre for Liver Disease, Réseau universitaire de santé, Toronto General Hospital, Toronto (Ontario)

${ }^{9}$ Centre Sandra Rotman, Université de Toronto, Toronto (Ontario)

*Correspondance: dena. schanzer@canada.ca 


\section{Introduction}

L'hépatite est reconnue comme étant un problème de santé publique à l'échelle internationale depuis des années (1). II y a plus de 15 ans, le gouvernement canadien a déterminé que le virus de I'hépatite $\mathrm{C}(\mathrm{VHC})$ contribue largement au fardeau croissant associé à la cirrhose, au carcinome hépatocellulaire $(\mathrm{CHC})$ et aux greffes de foie (2-3). En Ontario, on a déterminé que le VHC est la maladie infectieuse responsable du plus lourd fardeau en matière d'années de vie ajustées en fonction de la santé (4). Une étude de modélisation canadienne a estimé que la prévalence des cas de virémie par le VHC a atteint son apogée entre 2003 et 2013. Elle a également prédit que la prévalence de l'hépatopathie avancée augmenterait jusqu'en 2030, avec le vieillissement de la population infectée (5). Une autre étude canadienne basée sur les données relatives aux hospitalisations a déterminé qu'une grande partie de ce fardeau croissant était attribuable à la cohorte des baby-boomers. L'étude a par ailleurs révélé que le nombre d'hospitalisations attribuables aux hépatopathies chroniques associées au VHC avait augmenté de 6,0 \% par année entre 2004 et 2010 (6). En 2010, les taux $d$ 'hospitalisations étaient les plus élevés au sein des cohortes nées entre 1950 et 1959 et l'on projetait une augmentation de 1,5 fois le taux de 2010 lorsque la cohorte approcherait l'âge de 70 ans, de 2025 à 2035 (6).

Les antiviraux à action directe (AAD) de première génération sont entrés sur le marché canadien en 2012. Ces médicaments produisaient des taux élevés de réponse virologique soutenue et offraient l'espoir d'inverser la tendance concernant la prévalence de l'hépatopathie avancée. Leur tolérabilité était toutefois problématique et leur adoption est restée relativement limitée.

En 2014, une deuxième génération d'AAD très efficaces est entrée sur le marché. Ces nouveaux agents procuraient des taux de guérison de plus de $95 \%$ à l'égard de tous les génotypes principaux grâce à un traitement de courte durée (de 8 à 24 semaines) qui était bien toléré. Ces AAD de deuxième génération sont maintenant considérés comme étant une percée médicale majeure ayant révolutionné le traitement des infections par le VHC, ce qui a stimulé leur adoption et ouvert la possibilité d'une réduction importante de la morbidité et de la mortalité liées au VHC (7).

Encouragée en grande partie par les améliorations apportées au traitement contre les infections par le VHC, l'Organisation mondiale de la Santé (OMS) a appelé à l'élimination de I'hépatite virale en tant que menace pour la santé publique (7). L'OMS a fixé l'objectif à une réduction de $65 \%$ de la mortalité (comparativement au niveau de 2015) d'ici 2030 et recommande une surveillance continue de manière à ce que les gouvernements nationaux puissent évaluer s'ils sont en voie d'atteindre cet objectif ambitieux (7).

Il faudra attendre encore un certain nombre d'années avant que les données sur la mortalité ne soient disponibles, mais la surveillance des taux d'hospitalisation est une méthode d'évaluation viable qui offre même plusieurs avantages par rapport aux données sur la mortalité. En effet, les données sur les hospitalisations sont accessibles plus rapidement que celles sur la mortalité. Par ailleurs, on estime que les bases de données sous-estiment le nombre de décès dont la cause sous-jacente est liée au VHC (8). De plus, les données sur la mortalité ont une puissance statistique limitée (en raison du petit nombre de décès) qui retarde la détection des variations.

Cela dit, il faut tenir compte du fait que la réduction à court terme du fardeau de la maladie à l'échelle de la population que permettent les nouveaux traitements pourrait ne pas suffire à contrebalancer l'augmentation des taux d'hospitalisations qui est prévue (6). Trois aspects liés à l'évolution de la maladie expliquent ce déficit : premièrement, la réduction à court terme du risque d'hospitalisation devrait être principalement limitée au traitement des patients présentant une cirrhose compensée qui courent un risque de décompensation. Deuxièmement, le traitement des patients qui ne sont pas atteints d'une hépatopathie avancée préviendra l'évolution de la cirrhose à long terme. Finalement, l'état des patients atteints d'une hépatopathie avancée (en particulier ceux qui présentent une cirrhose décompensée ou un $\mathrm{CHC}$ ) pourrait ne pas connaître une forte amélioration après une réponse virologique soutenue, ce qui pourrait produire des effets limités ou nuls sur les taux d'hospitalisation (9-10).

Au Canada, l'étude menée en 2014 par Schanzer et ses collaborateurs a établi les projections de référence en matière d'hospitalisations liées aux infections par le VHC et aux hépatopathies chroniques en fonction des tendances observées avant l'introduction des AAD (6). Cette étude a révélé que l'augmentation du risque d'hospitalisation au sein de chaque cohorte de naissance (les cohortes étant divisées par tranche de cinq ans) était principalement liée à l'âge et que la stratification des cohortes en tranches de cinq ans était requise pour contrôler les différences sur le plan de l'exposition au VHC.

L'objectif de la présente étude était d'utiliser les projections qui avaient déjà été établies à titre de référence (situation hypothétique sans changement), de les comparer au nombre réel d'hospitalisations liées aux infections par le VHC et aux hépatopathies chroniques, et de documenter l'adoption du traitement antiviral à l'échelle de la population.

\section{Méthodologie}

\section{Sources de données}

Pour la période entre avril 2004 et mars 2017, les dossiers de sortie d'hôpital des patients qui avaient été admis à un hôpital de soins actifs et qui avaient reçu un code de diagnostic d'infection par le VHC (code de diagnostic B18.2 de I'ICD-10), d'hépatopathie chronique (K70-K77, R18) ou de CHC (C22), peu importe le champ diagnostique, ont été extraits de la Base de données sur les congés des patients (BDCP) de l'Institut canadien d'information sur la santé (ICIS) (11). Les champs contenant des renseignements sur l'âge, la date de sortie, le décès des patients hospitalisés et les cas de $\mathrm{CHC}$ ont été retenus pour l'analyse. Comme le Québec ne contribue pas à la BDCP, la base de données ne recueille les données que d'environ $75 \%$ de l'ensemble des hospitalisations pour soins actifs au Canada. Les dénominateurs de la population se basent sur les estimations de Statistique Canada (12). En septembre 2017, le nombre de nouvelles ordonnances pour un traitement anti-VHC qui ont été 
exécutées entre septembre 2011 et août 2017 a été obtenu de la base de données CDH CompuScript d'IQVIA (anciennement IMS Health) (13) et catégorisées par mois, par année, par province et par groupe d'âge. Cette base de données a été créée grâce à une enquête par panel menée auprès de plus de 5700 pharmacies (ce qui représente plus de $60 \%$ de toutes les pharmacies de détail au Canada). Les produits qui ont été utilisés pour traiter les infections par le VHC figurent à l'annexe 1. Les projections de référence en matière de taux d'hospitalisations par cohorte de naissance sont tirées de l'étude de 2014 menée par Schanzer et ses collaborateurs (6).

\section{Analyse des données}

\section{Tendances annuelles}

Pour évaluer les répercussions sur une base annuelle, la comparaison (nombre annuel réel d'hospitalisations par rapport au scénario de référence) a été limitée aux cohortes de naissance pour lesquelles il y avait suffisamment de données pour estimer les projections de référence (c.-à-d. aux personnes nées entre 1925 et 1989, mais âgées de moins de 90 ans). Les intervalles de confiance (IC) à $95 \%$ correspondent à l'écart-type de la différence entre les valeurs réelles et les prédictions faites avant l'introduction des AAD (de 2004 à 2011). Le rapport entre le nombre annuel d'hospitalisations et la projection de référence pour la même année a également été calculé. L'hypothèse voulait que ce rapport soit d'environ 1,0 pendant la période précédant l'introduction des $A A D$, et qu'il commence à baisser lorsque les $A A D$ de première et de deuxième génération ont commencé à réduire le risque d'hospitalisation. L'analyse de régression logistique a permis d'évaluer les tendances en matière de taux de mortalité chez les patients hospitalisés et de proportion des hospitalisations attribuables au $\mathrm{CHC}$.

\section{Groupes d'âge}

Pour évaluer les répercussions du traitement par groupe d'âge, le nombre d'hospitalisations associées à l'infection par le VHC et à l'hépatopathie chronique observé dans chaque cohorte de naissance (tranches de cinq ans) en 2016 à 2017 (selon la BDCP) a été comparé aux projections de référence pour l'exercice 2016-2017. L'âge moyen au moment de I'hospitalisation a été calculé pour chaque année et cohorte de naissance. Les barres d'erreur correspondant aux IC à $95 \%$ se fondent sur la distribution de Poisson généralement utilisée pour les données chiffrées.

\section{Nouvelles ordonnances}

Les quantités de nouvelles ordonnances émises par mois au Canada ont été illustrées en série chronologique. Le rapport des nouvelles ordonnances sur les hospitalisations a été calculé par année civile et par groupe d'âge à l'aide des données d'IQVIA. Ce rapport ne tient pas compte des nouvelles ordonnances émises au Québec.

\section{Résultats}

\section{Tendances annuelles}

Durant la période précédant l'introduction des AAD, le nombre annuel d'hospitalisations liées aux infections par le VHC et aux hépatopathies chroniques s'approchait des projections (figure 1 : comparer la courbe rouge à la courbe bleue). Une réduction significative sur le plan statistique a d'abord été observée en 2014-2015 (comparer la courbe verte à la courbe bleue). La réduction la plus prononcée (32\%; IC à $95 \%$ : de 27 à 37 \%) s'est produite en 2016 à 2017, lorsque le nombre d'hospitalisations liées aux infections par le VHC et aux hépatopathies chroniques était de 2538 , comparativement à la projection de 3740 .

\section{Figure 1 : Tendances dans le nombre annuel d'hospitalisations liées aux infections par le virus de I'hépatite $C$ et aux hépatopathies chroniques entre 2004-2005 et 2016-2017 au Canada ${ }^{a}$}

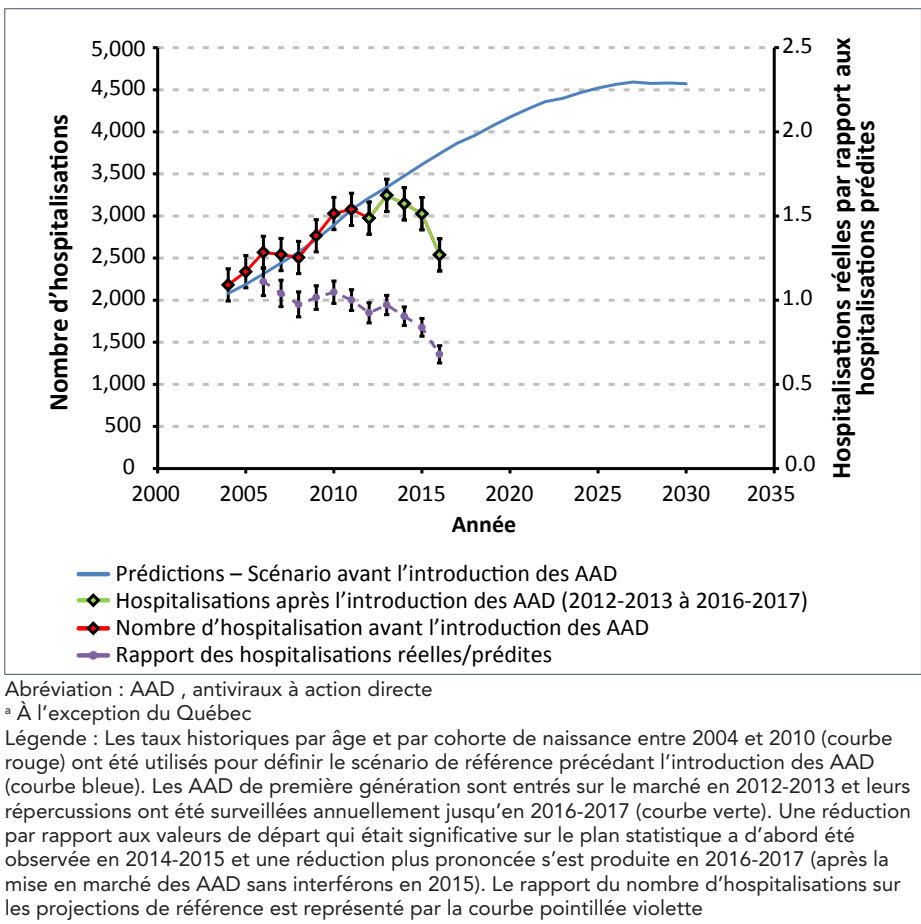

Chez les patients dont l'hospitalisation était liée à une infection par le VHC ou à une hépatopathie chronique, la mortalité a baissé de 15,7 \% en 2003 à 13,0 \% en 2016 (voir le tableau 1), ce qui correspond à une variation annuelle moyenne en pourcentage (VAMP) de $-1,2 \%$ (IC à $95 \%$ : de $-2,0$ à $-0,4 \%$ ). Après le contrôle du vieillissement de la cohorte de patients dont I'hospitalisation est liée à l'infection par le VHC, le taux de réduction devient plus prononcé (VAMP $=-1,9 \%$; IC à $95 \%$ : de $-2,6$ à $-1,1 \%$ ). Parmi les hospitalisations liées aux infections par le VHC et aux hépatopathies chroniques, la proportion de patients ayant reçu un diagnostic de $\mathrm{CHC}$ a augmenté de $11 \%$ 
à $25 \%$, ce qui correspond à une VAMP de 6,4 \% (IC à $95 \%$ : de 5,0 à 7,9 \%). La VAMP après le contrôle du vieillissement est de $4,0 \%$ (IC à $95 \%$ : de 2,9 à 5,1\%). La tendance est toutefois plus évidente pendant la période de référence (de 2003 à 2010), durant laquelle la VAMP est de 6,6 \% (IC à $95 \%$ : de 4,8 à 8,6\%).

Tableau 1 : Nombre d'hospitalisations liées aux infections par le virus de l'hépatite $C$ et aux hépatopathies chroniques par exercice financier, de 2003-2004 à 2016-2017, au Canada (tous âges compris)

\begin{tabular}{|r|r|r|r|r|r|}
\hline Année & $\begin{array}{r}\text { Hospitalisations } \\
(\mathbf{n})\end{array}$ & Décès $(\mathbf{n})$ & $\begin{array}{c}\text { Taux de } \\
\text { mortalité }(\%)\end{array}$ & $\begin{array}{c}\text { Cas de } \\
\mathrm{CHC}(\mathbf{n})\end{array}$ & $\begin{array}{c}\text { CHC } \\
(\%)\end{array}$ \\
\hline $2003-04$ & 2115 & 331 & 15,7 & 226 & 10,7 \\
\hline $2004-05$ & 2182 & 334 & 15,3 & 264 & 12,1 \\
\hline $2005-06$ & 2338 & 341 & 14,6 & 321 & 13,7 \\
\hline $2006-07$ & 2569 & 421 & 16,4 & 388 & 15,1 \\
\hline $2007-08$ & 2542 & 395 & 15,5 & 420 & 16,5 \\
\hline $2008-09$ & 2527 & 404 & 16,0 & 438 & 17,3 \\
\hline $2009-10$ & 2774 & 384 & 13,8 & 551 & 19,9 \\
\hline $2010-11$ & 3041 & 415 & 13,6 & 594 & 19,5 \\
\hline $2011-12$ & 3092 & 459 & 14,8 & 622 & 20,1 \\
\hline $2012-13$ & 2979 & 446 & 15,0 & 619 & 20,8 \\
\hline $2013-14$ & 3255 & 464 & 14,3 & 742 & 22,8 \\
\hline $2014-15$ & 3152 & 457 & 14,5 & 631 & 20,0 \\
\hline $2015-16$ & 3040 & 428 & 14,1 & 669 & 20,0 \\
\hline $2016-17$ & 2554 & 333 & 13,0 & 633 & 24,8 \\
\hline
\end{tabular}

Abréviations : année,exercice financier; $\mathrm{CHC}$, carcinome hépatocellulaire

À l'exception du Québec

- Proportion de patients dont l'hospitalisation était liée à l'infection par le virus de l'hépatite $C$ ou à l'hépatopathie chronique et qui avaient un diagnostic de carcinome hépatocellulaire

Source : Base de données sur les congés des patients de I'ICIS (11)

\section{Groupes d'âge}

La figure 2a compare les nombres réels d'hospitalisations pendant l'exercice 2016-2017 aux projections de référence. Pendant cette période, les baisses les plus fortes ont été enregistrées au sein des cohortes de naissance pour lesquelles les projections de référence étaient les plus élevées (de 1950 à 1954 et de 1955 à 1959). Le rapport des hospitalisations réelles sur les hospitalisations prévues (voir la figure $2 \mathrm{~b}$ ) montre que la baisse relative la plus importante (40\%) a été observée chez les patients nés avant 1960 (c.-à-d. chez les patients âgés de 55 ans et plus). À l'inverse, chez les patients de 45 ans et moins, le rapport de un suggère que le traitement n'a pas entraîné une réduction à court terme significative du risque d'hospitalisation.
Figure 2a : Nombre d'hospitalisations liées aux infections par le virus de l'hépatite $C$ et aux hépatopathies chroniques (présenté en fonction de l'âge ${ }^{a}$ ) observé en 2016-2017 au Canadab, comparativement aux estimations de référence

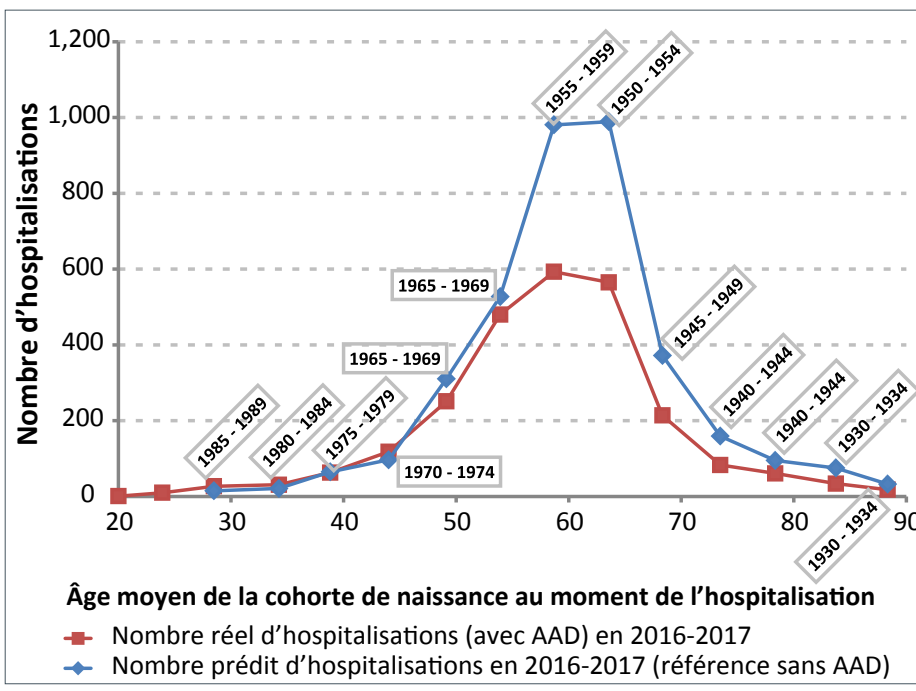

Figure $2 \mathrm{~b}$ : Nombre réel d'hospitalisations (en fonction de l'âge ${ }^{a}$ ) observé en 2016-2017 au Canada ${ }^{b}$ par rapport aux projections de référence établies avant l'introduction des antiviraux à action directe

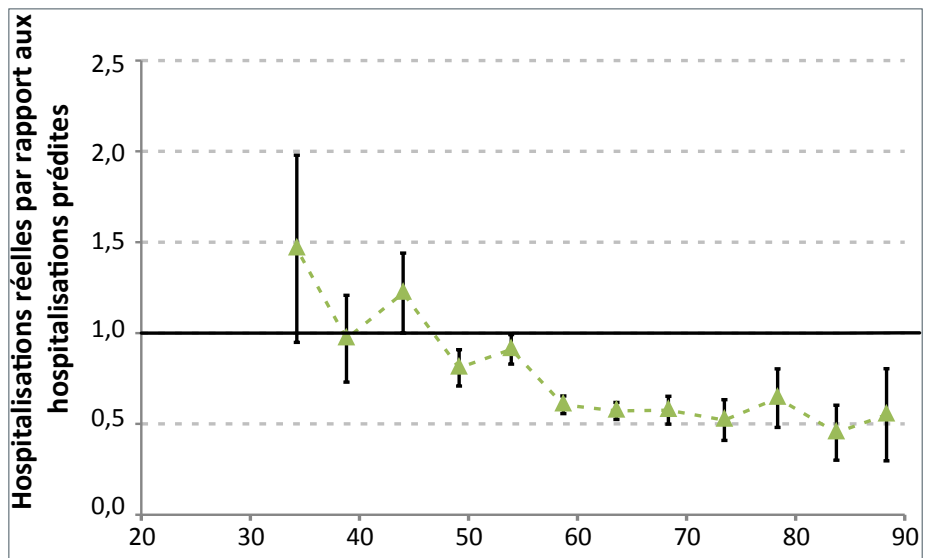

Âge moyen de la cohorte de naissance au moment de l'hospitalisation

- Rapport des hospitalisations réelles sur les hospitalisations prédites (référence sans $A A D$ )

Abréviations : $A A D$, antiviraux à action directe; $B D C P$, Base de données sur les congés des patients

a L'âge approximatif indiqué en abscisse correspond à l'âge moyen au moment de I'hospitalisation dans chaque cohorte de naissance (tranches de 5 ans). Les étiquettes apparaissant à la figure 2a correspondent aux cohortes de naissance. Les barres d'erreur du rapport entre les hospitalisations réelles et les valeurs de référence (figure $2 b$ ) correspondent aux intervalles de confiance à $95 \%$ calculés pour les données chiffrées

b À l'exception du Québec

Source : Base de données sur les congés des patients (11) 


\section{Nouvelles ordonnances}

Peu après l'arrivée sur le marché des AAD de deuxième génération, les nouvelles ordonnances ont atteint un sommet (figure 3), passant de 5484 en 2012 à 17775 en 2015.

Figure 3 : Nombre mensuel de nouvelles ordonnances de traitement contre les infections par le virus de I'hépatite $\mathrm{C}$ au Canada

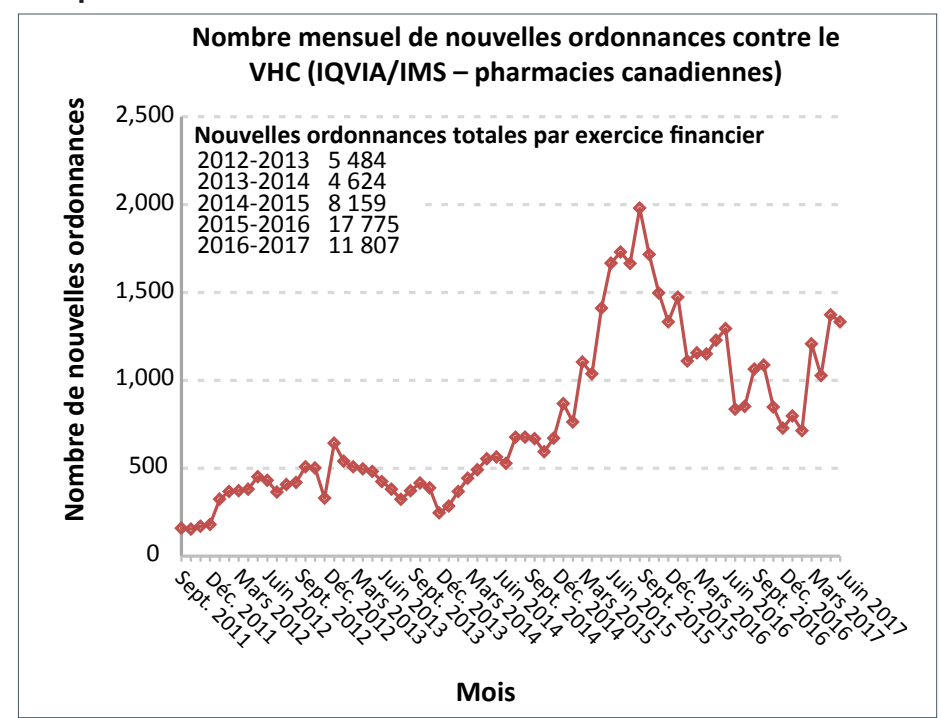

Abréviations : Rx, ordonnances; VHC, virus de l'hépatite $C$

Sources de données: CDH CompuScript d'IQVIA (anciennement IMS Health) (13)

Comme l'illustre le rapport des nouvelles ordonnances sur les hospitalisations pour chaque année et groupe d'âge (voir le tableau 2), la distribution en fonction de l'âge des nouvelles ordonnances était similaire à celle des hospitalisations, et ce, même si le groupe de patients traités est légèrement plus jeune que le groupe de patients hospitalisés.

Tableau 2 : Nombre de nouvelles ordonnances par rapport au nombre d'hospitalisations, pour tous les groupes d'âge, entre 2012 et 2016, au Canada

\begin{tabular}{|c|c|c|c|c|c|c|}
\hline \multirow[b]{2}{*}{$\begin{array}{l}\text { Année } \\
\text { civile }\end{array}$} & \multicolumn{5}{|c|}{ Groupe d'âge (années) } & \multirow[b]{2}{*}{ Total } \\
\hline & $19-29$ & $30-39$ & $40-49$ & $50-59$ & $\begin{array}{c}\text { Plus de } \\
60\end{array}$ & \\
\hline 2012 & 1,9 & 4,8 & 0,3 & 1,7 & 0,9 & 1,3 \\
\hline 2013 & 4,4 & 4,6 & 1,6 & 1,6 & 1,0 & 1,5 \\
\hline 2014 & 8,2 & 4,5 & 2,0 & 2,0 & 1,6 & 1,9 \\
\hline 2015 & 10,5 & 10,1 & 1,7 & 5,6 & 4,9 & 5,0 \\
\hline 2016 & 7,4 & 8,7 & 5,1 & 4,8 & 4,1 & 4,7 \\
\hline
\end{tabular}

à̀ l'exception du Québec

\section{Discussion}

Le nombre d'hospitalisations enregistrées au Canada en 2014-2015 et en 2015-2016 et liées aux diagnostics d'hépatopathies chroniques attribuables aux infections par le VHC est légèrement inférieur aux projections de référence. La réduction observée en 2016-2017 était modeste et survenait après une augmentation significative de l'adoption des traitements liée à l'arrivée sur le marché des AAD de deuxième génération (en 2015). Cette réduction est néanmoins impressionnante, puisque les projections prévoyaient une augmentation du fardeau. En utilisant les projections de référence obtenues d'une autre étude, il a été possible d'observer les premières phases de l'allègement du fardeau lié aux infections par le VHC et aux hépatopathies chroniques dans chaque groupe d'âge. Les personnes présentant une infection par le VHC qui sont nées avant 1960 (c.-à-d. âgées de 55 ans et plus en 2016) semblent avoir obtenu les bienfaits à court terme maximaux du traitement par les $A A D$, car une réduction de $40 \%$ du nombre d'hospitalisations (par rapport aux projections de référence) a été observée dans ce groupe en 2016. Les baisses les plus fortes se sont produites au sein des cohortes de naissance pour lesquelles les projections de référence étaient les plus élevées (de 1950 à 1954 et de 1955 à 1959). Malgré l'adoption proportionnellement plus importante du traitement par les $A A D$, aucune variation importante n'a été observée dans les cohortes plus jeunes, probablement en raison du stade de la maladie chez les patients recevant le traitement. Par exemple, les patients recevant le traitement dans les premiers stades de la fibrose courraient un très faible risque à court terme d'hospitalisation pour hépatopathie avancée, et les bienfaits les plus importants apparaîtraient à plus long terme dans ce groupe.

Une baisse du taux de mortalité chez les patients hospitalisés et une hausse de la proportion d'hospitalisations liées au CHC ont été observées sur la durée totale de l'étude (de 2003 à 2016). Malgré une certaine controverse concernant la possibilité que les $A A D$ entraînent une augmentation du risque de $\mathrm{CHC}$, des données plus récentes montrent clairement que la clairance du virus associée aux AAD réduit, sans l'éliminer, le risque de $\mathrm{CHC}$, d'une manière similaire à ce que procure la réponse virologique soutenue induite par l'interféron (14). Par contre, une forte augmentation de la mortalité et du risque de $\mathrm{CHC}$ liés à l'âge a été remarquée chez les patients présentant une infection chronique par le VHC (10). Les données émergentes confirment qu'une réponse virologique soutenue prévient la décompensation hépatique chez les patients atteints d'une cirrhose et améliore la fonction hépatique chez ceux qui reçoivent un traitement après une décompensation initiale; toutefois, le risque de $\mathrm{CHC}$ demeure une grave préoccupation (9). Les diminutions récentes du nombre de décès de patients hospitalisés et du nombre d'hospitalisations liées au $\mathrm{CHC}$ sont encourageantes, mais elles sont trop faibles et s'étendent sur une période trop courte pour permettre une évaluation de la signification statistique des répercussions des AAD sur la mortalité et les CHC.

\section{Points forts et limites}

Le principal point fort de la présente étude est la comparaison des données relatives aux hospitalisations aux projections de référence afin de fournir une évaluation plus rapide des répercussions des $A A D$ que ne le permettraient les statistiques de l'état civil (8). En outre, le grand nombre d'événements enregistrés chaque année fournit la puissance statistique nécessaire pour détecter les répercussions plus rapidement que les données sur la mortalité. La projection de référence correspondant à l'absence hypothétique d'un AAD (6) permettait également une évaluation plus précise des répercussions des nouveaux traitements que ne l'aurait permis une simple analyse des tendances. En effet, cette approche a permis de 
contrôler la variation de l'exposition aux infections par le VHC dans différences cohortes de naissance. En raison de la lente évolution de la maladie, le nombre d'hospitalisations précoces était un bon paramètre de prévision du nombre d'événements futurs. Par ailleurs, cette approche n'a pas nécessité les nombreux points de données, estimations et hypothèses qui sont requis pour modéliser toute la complexité de l'évolution de la maladie. Finalement, l'utilisation des valeurs hypothétiques de référence (plutôt que des valeurs historiques) à titre de comparateur a permis d'évaluer les bienfaits précoces potentiels de l'intervention et du contrôle pour l'augmentation prévue du fardeau.

II y a toutefois plusieurs limites dont il faut tenir compte. Les nombres d'hospitalisations liées au CHC étaient trop peu élevés pour permettre l'évaluation de tendances en la matière et aucune estimation de référence facilement accessible ne permettait de tenir compte du fardeau sous-jacent croissant. Comme il s'agissait d'une étude à l'échelle de la population, il n'y avait aucun renseignement propre aux patients (par exemple, les antécédents de la maladie, les soins administrés au patient et la catégorie d'exposition) qui aurait pu aider à raffiner les projections concernant le fardeau futur sous divers scénarios d'intensification de l'adoption du traitement et du dépistage. Aussi, puisque la présence d'un code diagnostique d'infection par le VHC ou d'hépatopathie chronique (tous champs diagnostiques confondus) dans la BDCP servait à repérer les hospitalisations liées à ces problèmes de santé, il existe un risque de biais attribuable au sous-dénombrement. En effet, il est probable que le diagnostic d'infection par le VHC n'ait pas été inscrit au dossier de sortie d'hôpital de tous les patients hospitalisés qui avaient reçu un tel diagnostic. En outre, le nombre de nouvelles ordonnances ne correspond pas nécessairement au nombre de cycles de traitement administrés, car les médecins pourraient ne pas avoir inscrit tous les renouvellements nécessaires pour l'ensemble du traitement sur une seule ordonnance. De plus, la qualité des données sur les ordonnances obtenues d'IQVIA n'a pas été entièrement évaluée. Malheureusement, comme le Québec ne contribue pas à la BDCP, les résultats relatifs aux hospitalisations ne s'appliquent pas à l'échelle nationale. Bon nombre de ces limites pourraient être levées grâce à des projets de couplage de données.

\section{Prochaines étapes}

Grâce à un traitement éventuellement curatif, la recherche est passée de la mesure et de la prédiction du fardeau de la maladie à la documentation des répercussions du traitement sur les résultats à long terme. Dans le contexte d'un fardeau croissant, les difficultés liées à la surveillance des répercussions des AAD à l'échelle de la population demeurent, de même que les préoccupations relatives à l'accès au traitement et au fardeau du CHC associé au VHC $(15,16)$ ainsi que les questions sur les répercussions d'une réponse virologique soutenue sur le futur fardeau du $\mathrm{CHC}(9,10,14)$. La réduction des cirrhoses compensées et décompensées et des $\mathrm{CHC}$ devrait pouvoir être détectée bien avant la baisse du taux d'hospitalisations, et c'est pourquoi elle constituerait un meilleur indicateur de la progression si ces données étaient disponibles en temps opportun. Une étude canadienne a utilisé les données liées à la santé concernant les décès pour caractériser les patients qui développaient une cirrhose décompensée ou un $\mathrm{CHC}$ attribuables au VHC entre 2007 et 2011 (16). Samji et ses collaborateurs ont défini un diagnostic tardif comme étant un diagnostic d'infection par le $\mathrm{VHC}$ reçu au cours des deux années précédant le diagnostic de cirrhose décompensée ou de $\mathrm{CHC}$ (16). Ils ont révélé que la proportion de diagnostics tardifs était considérablement inférieure pendant la période de l'étude (qui a précédé l'introduction des AAD) et que l'usage de drogues illicites (selon les dossiers médicaux) et les visites régulières chez le médecin étaient liés à une baisse du risque de diagnostic tardif (16). La capacité à observer ces tendances ainsi que les caractéristiques des personnes qui développent une cirrhose ou un $\mathrm{CHC}$ constituera un atout majeur dans la surveillance du progrès et l'orientation des efforts de la santé publique pour réduire encore davantage le fardeau de la maladie.

Les chercheurs associés au Réseau Canadien sur l'hépatite C (CanHepC) (17) mènent un certain nombre d'études supplémentaires qui visent à lier les cohortes de personnes ayant un diagnostic d'infection par le VHC aux autres bases de données administratives en santé (15)(16). La création de tels liens requiert diverses approbations ainsi que des dispositifs de protection de la confidentialité des patients. Bien que le partage véritable des données soit impossible, ces chercheurs prévoient coordonner les définitions utilisées dans les provinces de manière à ce que les résultats soient comparables. Des progrès importants ont été réalisés en Ontario (à l'Institut de recherche en services de santé), en Colombie-Britannique et au Québec et des statistiques additionnelles (liées à la manifestation tardive et à la série de soins administrés aux patients ayant un diagnostic d'infection par le VHC) viendront plus tard. Des recherches préliminaires ont comparé le nombre de nouvelles ordonnances obtenu de I'IQVIA Institute for Human Data Science aux données préliminaires concernant le nombre de cycles d'AAD administrés en Colombie-Britannique; elles ont révélé qu'un seul cycle de traitement est associé à environ 1,4 nouvelle ordonnance (communication personnelle, NZ Janjua, le 7 février 2018).

\section{Conclusions}

Une légère réduction des hospitalisations liées aux infections par le VHC et aux hépatopathies chroniques a été observée au Canada après l'arrivée des AAD de deuxième génération. Toutefois, le fardeau des hépatopathies associées aux infections chroniques par le VHC demeure élevé et l'évolution de la maladie jusqu'au CHC (après une réponse virologique soutenue chez les patients présentant une cirrhose) reste inquiétante. Comme il est préférable de traiter les patients atteints d'une infection chronique par le VHC avant l'apparition de la cirrhose, il faudra sûrement attendre de nombreuses années avant de constater la totalité des bienfaits associés au traitement par les AAD. Les tendances observées concordent avec les données émergentes indiquant qu'il est possible d'administrer un traitement efficace et sécuritaire aux patients présentant une hépatopathie très avancée. La reconnaissance et la surveillance des principaux indicateurs (comme le stade de la maladie lors de la réponse virologique soutenue) et les mesures relatives à la série de soins seront utiles pour la surveillance des progrès réalisés vers l'élimination du fardeau associé au VHC. Les bases de données administratives sur la santé créées pour surveiller le fardeau des maladies dans la nouvelle ère thérapeutique devraient fournir plus de données en permettant de faire des 
liens entre les antécédents thérapeutiques, l'évolution de la maladie et les résultats individuels.

\section{Déclaration des auteurs}

D. S. - Conceptualisation; méthodologie; rédaction - ébauche initiale; validation, analyse statistique

J. A. - Rédaction - révision et édition

M. G. R. - Rédaction - révision et édition

L. P. - Rédaction - révision et édition

K. T. - Rédaction - révision et édition

J. C. K. - Expertise clinique; données pour la validation et la comparaison avec d'autres études; rédaction - révision et édition N. J. - Données pour la validation et la comparaison avec d'autres études; rédaction - révision et édition

J. F. - Expertise clinique; données pour la validation et la comparaison avec d'autres études; rédaction - révision et édition

\section{Conflit d'intérêts}

J. F. a collaboré à des projets de recherche à AbbVie, Gilead, Janssen et Merck et a agi à titre de consultant pour Abbvie, Gilead et Merck.

\section{Remerciements}

Les auteurs souhaitent remercier l'Institut canadien d'information sur la santé (ICIS) ainsi que tous les intervenants ayant participé à la collecte et à la compilation de la Base de données sur les congés des patients (BDCP), ainsi que le programme d'accès et coordination des données de l'Agence de la santé publique du Canada pour avoir offert l'accès à cette base de données. Les auteurs souhaitent également exprimer leur reconnaissance pour la coopération de toutes les parties ayant participé à ces activités.

\section{Financement}

Ce travail a été réalisé grâce au soutien de l'Agence de la santé publique du Canada et du Réseau canadien sur l'hépatite C (subvention numéro NHC - 142832).

\section{Références}

1. Stanaway JD, Flaxman AD, Naghavi M, Fitzmaurice C, Vos T, Abubakar I, Abu-Raddad LJ, Assadi R, Bhala N, Cowie B, Forouzanfour MH, Groeger J, Hanafiah KM, Jacobsen KH, James SL, MacLachlan J, Malekzadeh R, Martin NK, Mokdad AA, Mokdad AH, Murray CJL, Plass D, Rana S, Rein DB, Richardus JH, Sanabria J, Saylan M, Shahraz S, So S, Vlassov V, Weiderpass E, Wiersma ST, Younis M, Yu C, El Sayed Zaki M, Cooke GS. The global burden of viral hepatitis from 1990 to 2013: findings from the Global Burden of Disease Study 2013. Lancet 2016 Sep;388(10049):1081-8. http://dx.doi. org/10.1016/S0140-6736(16)30579-7. PubMed (https://www. ncbi.nlm.nih.gov/pubmed/27394647)

2. Myers RP, Liu M, Shaheen AA. The burden of hepatitis $C$ virus infection is growing: a Canadian population-based study of hospitalizations from 1994 to 2004. Can J Gastroenterol 2008 Apr;22(4):381-7. http://dx.doi.org/10.1155/2008/173153. PubMed (https://www.ncbi.nlm.nih.gov/pubmed/18414713)

3. Zou S, Tepper M, El Saadany S. Prediction of hepatitis C burden in Canada. Can J Gastroenterol 2000 Jul-Aug;14(7):575-80. http://dx.doi.org/10.1155/2000/64270. PubMed (https://www.ncbi.nlm.nih.gov/pubmed/10978943)

4. Kwong JC, Ratnasingham S, Campitelli MA, Daneman N, Deeks SL, Manuel DG, Allen VG, Bayoumi AM, Fazil A, Fisman DN, Gershon AS, Gournis E, Heathcote EJ, Jamieson FB, Jha P, Khan KM, Majowicz SE, Mazzulli T, McGeer AJ, Muller MP, Raut A, Rea E, Remis RS, Shahin R, Wright AJ, Zagorski B, Crowcroft NS. The impact of infection on population health: results of the Ontario burden of infectious diseases study. PLoS One 2012;7(9):e44103. http://dx.doi.org/10.1371/journal. pone.0044103. PubMed (https://www.ncbi.nlm.nih.gov/ pubmed/22962601)

5. Myers RP, Krajden M, Bilodeau M, Kaita K, Marotta P, Peltekian K, Ramji A, Estes C, Razavi H, Sherman M. Burden of disease and cost of chronic hepatitis $\mathrm{C}$ infection in Canada. Can J Gastroenterol Hepatol 2014 May;28(5):243-50. http://dx.doi. org/10.1155/2014/317623. PubMed (https://www.ncbi.nlm.nih gov/pubmed/24839620)

6. Schanzer DL, Paquette D, Lix LM. Historical trends and projected hospital admissions for chronic hepatitis $C$ infection in Canada: a birth cohort analysis. CMAJ Open 2014 Jul;2(3):E139-44. http://dx.doi.org/10.9778/cmajo.20130087. PubMed (https://www.ncbi.nlm.nih.gov/pubmed/25295233)

7. Organisation mondiale de la Santé. Combating hepatitis B and $C$ to reach elimination by 2030: Advocacy brief. Geneva: WHO; 2016. http://www.who.int/hepatitis/publications/hepelimination-by-2030-brief/en/

8. Statistique Canada. Tableau 102-0521 - Décès, selon la cause, Chapitre I: Certaines maladies infectieuses et parasitaires (A00 à B99), groupe d'âge et sexe, Canada, CANSIM. Ottawa (ON): Statistique Canada; 2018. https://www150. statcan.gc.ca/t1/tbl1/fr/tv.action?pid=1310014101\&reque st_locale $=\mathrm{fr}$

9. van der Meer AJ, Feld JJ, Hofer H, Almasio PL, Calvaruso V, Fernández-Rodríguez CM Aleman S, Ganne-Carrié N, D'Ambrosio R, Pol S, Trapero-Marugan M, Maan R, Moreno-Otero R, Mallet V, Hultcrantz R, Weiland O, Rutter K, Di Marco V, Alonso S, Bruno S, Colombo M, de Knegt RJ, Veldt BJ, Hansen BE, Janssen HLA. Risk of cirrhosis-related complications in patients with advanced fibrosis following hepatitis C virus eradication. J Hepatol 2017 Mar;66(3):485-93. http://dx.doi.org/10.1016/j.jhep.2016.10.017. PubMed (https://www.ncbi.nlm.nih.gov/pubmed/27780714)

10. Waziry R, Hajarizadeh B, Grebely J, Amin J, Law M, Danta M, George J, Dore GJ. Hepatocellular carcinoma risk following direct-acting antiviral HCV therapy: A systematic review, meta-analyses, and meta-regression. J Hepatol 2017 Dec;67(6):1204-12. http://dx.doi.org/10.1016/j. jhep.2017.07.025 PubMed (https://www.ncbi.nlm.nih.gov/ pubmed/28802876)

11. Institut canadien d'information sur la santé. Métadonnées de la Base de données sur les congés des patients (BDCP). Ottawa (ON): Institut canadien d'information sur la santé; 2017. https://www.cihi.ca/fr/metadonnees-de-base-dedonnees-sur-les-conges-des-patients

12. Statistique Canada. CANSIM Tableau 051-0001 - Estimations de la population au 1er juillet, par âge et sexe, Canada, 
province ou territoire, annuelle, Ottawa (ON): Statistique Canada; 2016. https://www150.statcan.gc.ca/t1/tb/1/fr/tv.acti on?pid $=1710000501 \&$ request_locale $=\mathrm{fr}$

13. IQVIA Institute for Human Data Science. Canadian Drug Store \& Hospital Purchases Audit (CDH). 2017. http://www. imsbrogancapabilities.com/en/market-insights/cdh.html

14. Kanwal F, Kramer J, Asch SM, Chayanupatkul M, Cao Y, El-Serag HB. Risk of hepatocellular cancer in HCV patients treated with direct-acting antiviral agents. Gastroenterology 2017 Oct;153(4):996-1005.e1. http://dx.doi.org/10.1053/j. gastro.2017.06.012. PubMed (https://www.ncbi.nlm.nih.gov/ pubmed/28642197)

15. Alavi M, Janjua NZ, Chong M, Grebely J, Aspinall EJ, Innes $H$, Valerio HM, Hajarizadeh B, Hayes PC, Krajden M, Amin J,
Law MG, George J, Goldberg DJ, Hutchinson SJ, Dore GJ. The contribution of alcohol use disorder to decompensated cirrhosis among people with hepatitis $\mathrm{C}$ : an international study. J Hepatol 2018 Mar;68(3):393-401. http://dx.doi. org/10.1016/j.jhep.2017.10.019. PubMed (https://www.ncbi. nlm.nih.gov/pubmed/29107152)

16. Samji H, Yu A, Kuo M, Alavi M, Woods R, Alvarez M, Dore GJ, Tyndall M, Krajden M, Janjua NZ; BC Hepatitis Testers Cohort Team. Late hepatitis $B$ and $C$ diagnosis in relation to disease decompensation and hepatocellular carcinoma development. J Hepatol 2017 Nov;67(5):909-17. http://dx.doi.org/10.1016/j. jhep.2017.06.025. PubMed (https://www.ncbi.nlm.nih.gov/ pubmed/28684103)

17. CanHepC. Réseau Canadien sur I'Hépatite C. 2018. http:// www.canhepc.ca/fr

\section{Annexe 1}

Ordonnances pour un traitement contre l'infection par le VHC, par DIN du produit et combinaison de molécules (CDH CompuScript)

\begin{tabular}{|l|l|l|}
\hline \multicolumn{1}{|c|}{ Produit } & \multicolumn{1}{c|}{ Abréviation de la molécule ou de la combinaison } & \multicolumn{1}{c|}{ DIN } \\
\hline DAKLINZA & DACLATASVIR & 2444747,2444755 \\
\hline EPCLUSA & SOFOSBUVIR:VELPATASVIR & 2456370 \\
\hline GALEXOS & SIMÉPRÉVIR & 2416441 \\
\hline HARVONI & LÉDIPASVIR:SOFOSBUVIR & 2432226 \\
\hline HOLKIRA PAK & DASABUVIR:OMBITASVIR:PARITAPRÉVIR:RITONAVIR & 2436027 \\
\hline IBAVYR & RIBAVIRIN & $2425890,2425904,2439212$ \\
\hline INCIVEK & TÉLAPRÉVIR & 2371553 \\
\hline PEGASYS & PEGINTERFÉRON ALFA 2A & 2248077,2248078 \\
\hline PEGETRON & INTERFÉRON ALFA 2B:RIBAVIRIN & $2246026,2246027,2246028,2246029,2246030$ \\
\hline PEGETRON REDIPEN/CLEARCL & INTERFÉRON ALFA 2B:RIBAVIRIN & $2254581,2254603,2254638,2254646$ \\
\hline SOVALDI & SOFOSBUVIR & 2418355 \\
\hline TECHNIVIE & OMBITASVIR:PARITAPRÉVIR:RITONAVIR & 2447711 \\
\hline VICTRELIS & BOCÉPRÉVIR & 2370816 \\
\hline VICTRELIS TRIPLE & BOCÉPRÉVIR:PEGINTERFÉRON ALFA 2B:RIBAVIRIN & $2371448,2371456,2371464,2371472$ \\
\hline ZEPATIER & ELBASVIR:GRAZOPRÉVIR & 2451131 \\
\hline
\end{tabular}

